\title{
Review
}

\section{Complementary and Alternative Medicine Approaches for Pediatric Pain: A Review of the State-of-the-science}

\author{
Jennie C. I. Tsao and Lonnie K. Zeltzer \\ Pediatric Pain Program, Departments of Pediatrics, Anesthesiology, Psychiatry and Biobehavioral Sciences, \\ David Geffen School of Medicine at UCLA, Los Angeles, CA, USA
}

\begin{abstract}
In recent years, the use of complementary and alternative medicine (CAM) in pediatric populations has increased considerably, especially for chronic conditions such as cancer, rheumatoid arthritis and cystic fibrosis in which pain may be a significant problem. Despite the growing popularity of CAM approaches for pediatric pain, questions regarding the efficacy of these interventions remain. This review critically evaluates the existing empirical evidence for the efficacy of CAM interventions for pain symptoms in children. CAM modalities that possess a published literature, including controlled trials and/or multiple baseline studies, that focused on either chronic or acute, procedural pain were included in this review. The efficacy of the CAM interventions was evaluated according to the framework developed by the American Psychological Association (APA) Division 12 Task Force on Promotion and Dissemination of Psychological Procedures. According to these criteria, only one CAM approach reviewed herein (self-hypnosis/guided imagery/relaxation for recurrent pediatric headache) qualified as an empirically supported therapy (EST), although many may be considered possibly efficacious or promising treatments for pediatric pain. Several methodological limitations of the existing literature on CAM interventions for pain problems in children are highlighted and future avenues for research are outlined.
\end{abstract}

Keywords: complementary medicine - alternative medicine - pediatric pain - pain - children

\section{Introduction-Use of CAM for Pediatric Pain: What's the Evidence?}

Complementary and alternative medicine (CAM) has been defined as those interventions not generally provided by US hospitals and clinics, nor widely taught in medical schools (1). In children, estimates of CAM use have varied widely from as low as $2 \%$ (2) to as high as $20-30 \%(3,4)$. Comparisons across studies are complicated by several factors such as lack of consensus in the definition of CAM, the inclusion of different populations and variations in study methodology. Despite these difficulties, recent work has suggested that use of CAM is increasing substantially in pediatric populations (3). However, there have been no population-based studies describing reasons for using CAM in children, although prior research indicates that children with chronic conditions which may not

For reprints and all correspondence: Jennie C. I. Tsao, PhD, Pediatric Pain Program, Department of Pediatrics, David Geffen School of Medicine at UCLA, 10940 Wilshire Blvd., Suite 1450, Los Angeles, CA 90024, USA. Tel: +1-310-824-7667; Fax: +1-310-824-0012;

E-mail: jtsao@mednet.ucla.edu be responsive to conventional treatments appear to have especially high rates of CAM use (5). In accord, rates of CAM use among pediatric patients with chronic conditions such as cancer, rheumatoid arthritis and cystic fibrosis range from 30 to $70 \%(6,7)$. For many of these conditions, pain may be a significant problem. In the general population, chronic pain is among the main reasons for which CAM is used (8-10). One study reported that, in a representative US sample of adults, musculoskeletal disorders (e.g. osteoarthritis, back disorders and joint disorders) were among the top three precipitating reasons for visiting a CAM practitioner (9). Another nationally representative US study of adults reported that the highest condition-specific rates of CAM use were for neck (57\%) and back (47.6\%) problems (10).

The increased interest in CAM approaches for pain symptoms in the pediatric and general populations has focused attention on questions of safety and efficacy. Although case reports and non-controlled investigations may offer important preliminary information, for example regarding the acceptability of such interventions, rigorously conducted controlled studies are needed to determine whether CAM approaches can be

(C) The Author (2005). Published by Oxford University Press. All rights reserved.

The online version of this article has been published under an open access model. Users are entitled to use, reproduce, disseminate, or display the open access version of this article for non-commercial purposes provided that: the original authorship is properly and fully attributed; the Journal and Oxford University Press are attributed as the original place of publication with the correct citation details given; if an article is subsequently reproduced or disseminated not in its entirety but only in part or as a derivative work this must be clearly indicated. For commercial re-use, please contact journals.permissions@oupjournals.org 
considered safe and effective treatments for pain in children. An important framework to assist in the evaluation of efficacy was developed by the American Psychological Association (APA) Division 12 Task Force on Promotion and Dissemination of Psychological Procedures (referred to below as 'APA Task Force') $(11,12)$. For a treatment to be considered 'efficacious', there must be a minimum of two between-group experiments conducted by at least two independent research groups showing that the intervention is superior to a no-treatment control, an alternative treatment or a placebo; or that the intervention is equivalent to a previously established treatment (11). For a designation of 'possibly efficacious' only one between-group study that meets these criteria is sufficient. To be considered 'efficacious and specific', a treatment must be shown by at least two independent research groups to be superior to placebo or a previously established treatment in studies that controlled for non-specific effects (e.g. increased attention). Further requirements include: (i) use of a treatment manual or logical equivalent; (ii) a clearly delineated population with specified inclusion criteria treated for specific problems; (iii) use of reliable and valid outcome measures; and (iv) appropriate data analyses. Treatments meeting all these criteria may be considered empirically supported therapies (ESTs).

This review aims to evaluate the empirical evidence for the efficacy of CAM approaches for pediatric pain problems according to the guidelines proposed by the APA Task Force. Only those studies that included samples entirely comprised of participants aged 17 years and younger are reviewed. The particular CAM interventions discussed below are included because they possess an existing literature that includes either at least one multiple baseline design or at least one controlled trial. Thus, the list of CAM modalities reviewed herein is not exhaustive. Studies on CAM interventions for palliative care in children are not included in this review since a review of the extant literature found no published studies meeting the criteria of a multiple baseline or controlled trial. Within each CAM approach discussed below, studies are grouped according to whether the intervention was used for chronic or acute/ procedural pain; within each of these broad categories, studies for specific conditions (e.g. pediatric migraine) are grouped together. The discussion below also focuses on the methodological limitations of existing studies as well as some of the major difficulties with conducting outcome research for specific modalities.

\section{Acupuncture}

In the adult population, the effectiveness of acupuncture has received empirical support for various pain problems [e.g. headaches (13) and chronic back pain (14)]. Moreover, reports of serious adverse effects of acupuncture are rare $(15,16)$. Despite substantial evidence for its efficacy and safety in adults, there are very few published reports on acupuncture for treatment of pain in children. One possible reason for the paucity of work in younger populations may be the conventional view that children do not like or are afraid of needles
(17). Thus, not only do clinicians hesitate to recommend acupuncture because of concerns regarding its acceptability (17), but researchers may also be reticent to conduct acupuncture trials in younger samples due to concerns regarding patient enrollment and/or retention. Nevertheless, Kemper and colleagues (17) reported that in a sample of children referred to an acupuncturist for chronic pain problems (e.g. migraine headaches, endometriosis and reflex sympathetic dystrophy), $67 \%$ reported that acupuncture was a positive experience and $70 \%$ reported that it definitely helped their pain. Among parents, $60 \%$ stated that acupuncture was a positive experience and 59\% reported that it definitely helped their child's pain. Such results support the feasibility and acceptability of acupuncture for chronic pediatric pain problems. There were, however, some important limitations. First, most of the patients in this study were adolescents (median age $=$ 16 years). Secondly, only patients who were referred and actually went to the acupuncturist were interviewed. Because no information was available regarding the percentage of referred patients that refused acupuncture, the authors acknowledge that they may have overestimated the acceptability of treatment. They therefore recommended further prospective investigations, particularly in younger samples.

\section{Chronic Pain}

One such study by Zeltzer et al. (18) examined the feasibility and acceptability of a combined acupuncture and hypnotherapy package in 33 children, $6-18$ years of age $($ mean $=13.0)$, referred to a tertiary chronic pain clinic. Children were referred for a variety of problems including myofacial and migraine headaches (46\%), abdominal pain (21\%), fibromyalgia $(11 \%)$ and complex regional pain syndrome, type I, of an extremity (11\%). Treatment comprised six weekly sessions of acupuncture together with a 20 min hypnotherapy session conducted while the needles were in place. Only two patients refused the treatment, indicating high acceptability, and $>90 \%$ completed treatment. In addition, no adverse effects were reported. Both parents and children reported significant improvements in children's pain and functioning following treatment. These findings support the acceptability of a combined acupuncture/hypnotherapy intervention. However, because the acupuncture was part of a combined treatment package, the acceptability of acupuncture alone (i.e. without hypnotherapy) could not be determined. Moreover, this study did not include a control group and, therefore, conclusions regarding the efficacy of the combined acupuncture/ hypnotherapy package could not be drawn.

\section{Pediatric Migraine}

There has only been one randomized, controlled study on acupuncture in children with chronic pain. In this study by Pintov and colleagues, 22 patients aged 7-15 years with migraine headaches received either true acupuncture or placebo acupuncture (superficial needling) (19). The true acupuncture group $(n=12)$ was treated according to the principles of 
Traditional Chinese Medicine with needles inserted subdermally. In the placebo group $(n=0)$, needles of the same size were inserted in the stratum corneum. Children, as well their parents and the nurses who administered the pain measures, were all unaware of study group assignment. Both groups received 10 weekly treatment sessions and no children received prophylactic medications. Given that dysregulation of the endogenous opioid antinociceptive system has been proposed in migraine $(20,21)$, blood samples were taken to examine treatment effects on plasma panopioid acitivty and levels of $\beta$-endorphin. The results showed that the true acupuncture group had clear reductions in migraine frequency and severity. In addition, panopioid activity in plasma and $\beta$-endorphin levels rose significantly in the true acupuncture group. No such changes, however, were observed in the placebo group. Although the study employed a rigorous design, it should be noted that the sample sizes were relatively small. In addition, patients receiving medication were excluded from the study, even though many migraine patients are on regular, prophylactic and/or as needed medications. Thus, the study sample may not be representative of pediatric migraine patients. Also, no information on refusal rates was reported, so the acceptability of the treatment remains unknown. Finally, no follow-up data were presented so it is unclear whether treatment gains persisted across time. Nevertheless, these findings support the efficacy of acupuncture in the treatment of pediatric migraine.

Evidence from the Pintov et al. (19) study supports the designation of possibly efficacious for acupuncture in the treatment of pediatric migraine. For a range of chronic pediatric pain syndromes, existing evidence from Zeltzer et al. (18) suggests that acupuncture may be considered a promising intervention according to APA Task Force criteria. Thus, more research is required to establish the efficacy of acupuncture for chronic pain problems in children. No published studies to date have examined the use of acupuncture for acute pain in children.

\section{Biofeedback}

Several studies published since the 1980s have examined the effects of biofeedback (BFB) on pain in children, with the majority focused on pediatric migraine and a few on tension headache. The most frequently studied forms of BFB for head pain in children are skin temperature or thermal biofeedback (TBF; volitional handwarming), which has been used primarily for migraine, and electromyographic biofeedback (EMGBFB) from the frontalis (forehead) muscle (22), which has been used mainly for tension headaches. TBF typically involves monitoring visual and/or auditory feedback from a thermistor placed on the fingers. EMG-BFB involves monitoring visual and/or auditory feedback from electric impulses generated from the frontalis muscle.

\section{Pediatric Migraine}

In their comprehensive review of 15 studies, Hermann and Blanchard (22) maintain that TBF for pediatric migraine may be considered 'possibly efficacious' according to the APA Task Force criteria. According to the authors, TBF does not qualify for an EST, as it does in adults, because it has not shown effects superior to that of a credible placebo condition or alternative interventions. Since their review was published, one study (23) has compared TBF (HWB; handwarming) with an attention placebo ( $\mathrm{HCB}$; handcooling) and waiting list in 36 children (mean age $=12.8$ years) with pediatric migraine. The results indicated that a significantly greater proportion of the HWB group (53.8\%) achieved clinically significant improvement (i.e. $\geq 50 \%$ reduction in symptoms) compared with the HCB group (10\%) at post-treatment, and 3 and 6 month follow-ups. The waiting list group did not show significant changes. These findings (23) provide evidence in support of the efficacy of TBF in pediatric migraine. However, because TBF was part of an overall treatment package that included many other components (i.e. progressive muscle relaxation, imagery training of warm places and vasodilation, and instruction in deep breathing), it is not possible to attribute improvements to any single component. Thus, further work comparing TBF alone with a credible attention placebo, or an established treatment, is warranted to meet criteria for an EST. Also as Hermann and Blanchard (22) pointed out, although several TBF studies have shown maintenance of treatment gains for periods up to 1 year, the limited available data on the natural course of pediatric migraine suggest that alternative, non-specific factors (e.g. growing out of it) cannot currently be completely ruled out (24).

\section{Tension Headache}

Compared with pediatric migraine, relatively little empirical work has focused on the effects of BFB on tension headaches in children. In their review, Hermann and Blanchard (22) conclude that EMG-BFB may be considered a promising intervention if the APA Task Force criteria are applied 'rather leniently' (p. 154). Despite high success rates of $80-90 \%$ in the three existing studies (25-27), two of these studies were conducted by the same research group. Moreover, Hermann and Blanchard point out limitations of small cell sizes and large variation in treatment outcome. In addition to EMG$\mathrm{BFB}$, two other studies have examined BFB-assisted relaxation in children with tension headaches. Only one of these studies (28) used a randomized controlled design, comparing EMG-BFB with relaxation in 35 patients (aged 11-15 years). Results indicated that although the groups were equivalent at 1 month post-treatment, by 6 and 12 month follow-ups, the EMG-BFB group achieved significantly greater reductions in symptoms compared with controls. Thus, further work is needed for EMG-BFB to achieve EST status in treating childhood tension headaches.

TFB was recently examined in the treatment of tension headaches in five children (aged 8-14 years) using a multiple baseline design (29). Treatment consisted of six sessions with four devoted to TBF training and two additional followup/problem-solving sessions. Following treatment, all patients 
experienced clinically significant reductions in symptoms compared with baseline. Nevertheless, TBF was part of a treatment package that included guidelines for parent support of children's independent pain behavior management, and patients were allowed to use pain medication as needed. Thus, it appears that TBF holds promise as an intervention in childhood tension headaches, although further carefully controlled between-group studies are warranted.

\section{Other Pain Problems}

The use of BFB for the management of pain problems other than headache in children has rarely been studied. One possible reason is that the physiological response to be modified in the desired direction is less clear in disease-related (e.g. cancer) pain or other pain problems (e.g. recurrent abdominal pain) (22). One study reported moderate pain relief in eight children with juvenile arthritis who were treated with a package containing progressive muscle relaxation, EMB-BFB and TBF, as well as parent pain management training (30). In eight children with sickle cell disease, BFB-assisted relaxation, including EMG-BFB and TBF, significantly reduced pain and frequency of self-treated pain episodes but did not reduce the number of hospital-treated pain crises (31). It is possible that if psychological factors, such as perceived self-efficacy, are found to mediate treatment response to BFB rather than muscle tension per se, a greater emphasis on such self-control aspects rather than on relaxation may encourage use of BFB for childhood pains other than headache (22).

\section{Creative Arts}

Interventions involving the creative arts, including music therapy, art therapy (AT) and movement/dance therapy, have been the subject of case studies (32) and uncontrolled investigations (33) on the management of pain in pediatric populations, but there are only a handful of controlled studies. Many existing reports suffer from methodological limitations that make it difficult to draw definitive conclusions regarding efficacy. For example, in one case-controlled study on AT (34), children with leukemia (aged 2-14 years) administered an AT package were compared with children previously admitted to the unit who had not received AT; however, no standardized measures were used to assess outcome, and children's pain per se during medical procedures (e.g. lumbar puncture) was not measured. In addition, the AT package included seemingly unrelated interventions (e.g. visual imagination, medical play, repeated reading and dramatization) as well as structured and free drawing. Although the authors maintained that children given AT appeared to exhibit more cooperative behavior than non-AT children during medical procedures, it is unclear who conducted such evaluations and what standards were used to arrive at these conclusions.

The majority of controlled studies using the creative arts have involved some form of music intervention for acute, procedural pain; there are no published controlled investigations for chronic pain in pediatric samples. Whereas music therapy may involve either trained therapists who perform live or music interventions for procedural pain that primarily involve recorded music delivered via headphones, it is unclear whether these modalities can be clearly differentiated conceptually in terms of therapeutic mechanism(s). It appears that both such modalities function mainly as a form of distraction that indirectly influences the pain response. Distraction may facilitate habituation to painful stimuli (35), perhaps because engaging in an alternative, attentionally demanding task limits the capacity to process pain, thereby reducing pain sensitivity (36). It is not known whether music exerts any additional effects on pain above and beyond distraction. Moreover, it is unclear whether music functions as a superior distractor relative to other stimuli. The question of a suitable placebo condition for studies on music therapy is of particular importance since almost any type of auditory stimulation, even white noise, has some kind of distracting quality. Yet, as discussed below, very few studies have included a suitable distraction control condition.

\section{Procedural Pain}

In an early study, Fowler-Kerry and Lander (37) compared the following four conditions on injection pain in 200 children (aged 4.5-6.5 years): (i) music distraction (music played over headphones before and during injection); (ii) suggestion (verbal instructions that the experimenter would help the child during the injection); (iii) distraction plus suggestion; and (iv) two control groups (i.e. no intervention; headphones without music). Music distraction was superior to suggestion in reducing pain, but there was no incremental effect on pain when suggestion was added to music. It should be noted, however, that pain was assessed using a 4-point visual analog scale whose psychometric properties are not known. Moreover, the inclusion of an auditory distraction condition (e.g. white noise) would have strengthened the study design. More recently, another study (38) in 99 children (aged 3-6 years) found that those who listened to lullabies during immunization showed less behavioral distress than no intervention controls, although the groups did not differ on physiological responses or reported pain. One important limitation was that it was unclear whether children were randomly assigned to groups. Nonrandom assignment increases the likelihood that obtained group differences may be due to factors other than the treatment itself, even when non-random groups are matched on pre-intervention scores. Also, no information was provided regarding how the distress ratings were conducted; for example, it unclear whether raters were aware of group assignment.

Another study examined the effects of live music therapy for pain related to intravenous starts, venipunctures, injections and heel sticks in 20 pediatric patients aged 0-7 years and 20 children, matched for age and type of needle insertion who did not receive the intervention (39). The results indicated that the music group showed less behavioral distress than the control group during pre-needle and post-needle stages, although there 
were no group differences in distress during needle insertion. In addition, this study had several methodological limitations. It was unclear whether patients were randomly assigned to groups and whether raters of distress were aware of group assignment. Moreover, nurses and intravenous therapists in the control condition were instructed to 'maintain their normal modes of consolation or distraction', and is it not known whether this varied across study groups.

It should be noted that negative findings for music interventions have also been reported. One study examined the impact of music on anxiety, pain and disruptive behavior in 45 children (aged 4-6 years) during pediatric dental procedures (40). All children had two visits involving restorative dentistry with local anesthesia. The first visit was a baseline session; during the second visit, children were assigned to one of three conditions: upbeat music, relaxing music or no music. The results indicated no significant differences among the groups on parent- or child-reported anxiety, heart rate, cooperative behavior or child-rated pain. In a study that compared the effects of lidocaine-prilocaine emulsion (EMLA) with placebo emulsion and with music distraction on pain of intravenous cannulation in children aged 4-16 years, music was no better than placebo and both were less effective than EMLA (41). It should be noted however, that the EMLA and placebo were administered $1 \mathrm{~h}$ prior to cannulation whereas the music distraction was started just before the procedure. In another study (42), the effects of music were examined in 121 neonates undergoing unanesthetized circumcision randomly assigned to one of six groups: classical music, intrauterine sounds, pacifier, music and pacifier, intrauterine sounds and pacifier, or control (no intervention). This study is one of the few published works that compared the effects of music with that of other auditory stimuli. However, the results indicated that there were no significant differences between groups in pain reduction as indexed by behavioral and physiological measures during the invasive portions of the procedure.

In sum, despite a lack of rigorous research on other areas of the creative arts, there are at least a few well-controlled studies on the effects of music interventions on pain in pediatric populations. To date, however, there have been no controlled studies on the impact of music interventions in chronic pediatric pain populations, and this is an area that warrants further study. Music may be considered a promising intervention for procedural pain if the APA Task Force criteria are loosely applied. Future work should include appropriate placebo groups using auditory stimuli other than music to test the notion that music is superior to other auditory distractions. In addition, other potential factors that might modulate treatment outcome such as anxiety should be investigated.

\section{Herbal Medicine}

\section{Ear Pain}

Use of herbal medicines is popular in the general population (12), but there are very few published studies examining herbal medicine and pain in children. Two randomized, double-blind controlled trials by Sarrell and colleagues tested a naturopathic herbal extract (NHE) on ear pain associated with acute otitis media (AOM) $(43,44)$. In the first study, 103 children (aged 6-8 years) with AOM were randomly assigned to receive NHE $(n=61)$ or anesthetic ear drops (AE) $(n=42)$ on day 1 (at the clinic), and then at home on days 2 and 3. Both groups showed significant reductions in pain across time, with the NHE group reporting less pain on day 1 compared with the AE group. In the second study (44), 171 children (aged 5-8 years) were randomly assigned to one of four conditions: NHE alone, $\mathrm{NHE}+$ oral amoxicillin, $\mathrm{AE}$ alone or $\mathrm{AE}+$ oral amoxicillin. The drops were administered as in the previous study across 3 days. All groups showed significant decreases in pain, with significantly greater pain relief in patients given AE only on days 2 and 3, compared with those given $\mathrm{AE}$ and antibiotics. Nevertheless, the authors point out that study variables accounted for only $22 \%$ of the variance in pain reduction, suggesting that the remaining variance may be explained by other factors such as passage of time alone. Prior reports have indicated spontaneous recovery rates of $70-90 \%$ in children with $\mathrm{AOM}(45,46)$. In addition, children younger than age 5 were excluded from the study due to limited ability to report pain accurately, even though AOM is more prevalent in 2-3 year olds.

Despite these limitations, it is noteworthy that the effects of naturopathic extract in reducing ear pain were equivalent to that of anesthetic drops and also that antibiotics did not improve the effects of the extract on ear pain. The naturopathic extract used in these two studies has been found to have analgesic, antiinflammatory, hygroscopic and occlusive effects, as well as anti-infective properties (47). In addition, there were no adverse effects reported in either study. Strictly speaking, the APA Task Force criteria require the naturopathic extract to show superior effects to the anesthetic drops for the designation of efficacious. It is possible that the naturopathic extract would have demonstrated better effects than a placebo although ethical considerations may preclude withholding of active treatment. Nevertheless, given the high rate of spontaneous recovery from AOM, such a trial may be conceivable. In sum, using a conservative reading of the APA Task Force criteria, the naturopathic extract may be considered a promising treatment for ear pain related to AOM in children. There does not appear to be any published research on the use of herbal medicine for other pediatric pain problems, acute or chronic.

\section{Functional Abdominal Pain}

There has been a single randomized control trial investigating the effects of peppermint oil compared with placebo for pain and related symptoms in 50 children (ages 8-12 years) with irritable bowel syndrome (48). By the end of the 2 week trial, a significantly greater proportion of the treatment group (71\%) reported improvements in severity of symptoms compared with controls (43\%). Analyses of daily patient diaries revealed that the mean severity of pain symptoms was significantly lower than that of the placebo group. No adverse effects were reported. 
In a subsequent review of treatments for recurrent abdominal pain (RAP) (49), it was reported that the effect size (i.e. the standardized mean difference between treatment and control groups) for this study on child-reported daily pain symptoms was 2.25 , indicating a large effect of treatment. In fact, of the four double-blinded placebo-controlled trials reviewed for RAP, peppermint oil showed that it was a more beneficial treatment for pain than fiber, and the pharmaceuticals famotidine $\left(\mathrm{H}_{2}-\right.$ receptor antagonist) and pizotifen (serotonin antagonist) (49). However, until these findings are replicated in an independent research group, peppermint oil may be considered possibly efficacious for functional abdominal pain in children.

\section{Homeopathy}

According to the National Center for Complementary and Alternative Medicine (NCCAM; http://nccam.nih.gov/health/ homeopathy/index.htm website viewed March 12, 2005), homeopathy seeks to stimulate the body's defense mechanisms and processes so as to prevent or treat illness. Treatment involves administering very small doses of substances called remedies that, according to homeopathy, would produce the same or similar symptoms of illness in healthy people if they were administered in larger doses. Treatment in homeopathy is individualized (tailored to each person). Homeopathic practitioners select remedies according to a total picture of the patient, including not only symptoms but lifestyle, emotional and mental states, and other factors. Thus, homeopathy is an alternative medical system that may use natural substances including herbs and vitamins. Whereas herbs and other natural substances may be administered outside the homeopathic approach, the studies below refer to the system of homeopathy which involves diagnosis, classification and treatment.

Homeopathic treatment has been the subject of very few controlled studies for pediatric pain. The most rigorous published study to date examined the effects of homeopathic medicine on ear pain associated with AOM in 75 children aged 18 months to 6 years (50). Using a double-blind randomized design, half of the children were given active, individualized homeopathic medicine and the other half received placebo. Treatment failure was defined as ear pain and/or fever $\left(\geq 38.0^{\circ} \mathrm{C}\right.$ orally) at any time after the first $48 \mathrm{~h}$ of treatment, or severe ear pain (e.g. crying from pain) and/or fever $\left(\geq 39.0^{\circ} \mathrm{C}\right.$ orally) after the first $24 \mathrm{~h}$. Results indicated fewer treatment failures in the homeopathy group compared with the control group, but these differences were not statistically significant. However, daily symptom diary scores (i.e. pain, fever, irritability, appetite, energy level, sleep and concurrent respiratory tract symptoms) completed by parents showed significant improvement in the homeopathic group compared with controls after 24 and $64 \mathrm{~h}$ of treatment. However, the diary scores were composites of all the symptoms and therefore it is not known what effects were specific to pain. In addition, the authors pointed out that an inherent methodological problem with any clinical trial involving homeopathy is that homeopathic treatment requires individualization of medication to the patient, and that therefore more than one medicine must necessarily be used. Thus, they reasoned that giving the same homeopathic medicine to all patients would not be a valid test of homeopathy. In sum, given that it is not possible from the current findings to isolate the impact of homeopathy on pain, the designation of 'promising' is given. There are currently no published reports on the use of homeopathy for other pain problems in children.

\section{Hypnosis}

Since the 1980s, numerous studies have investigated the application of hypnosis to pain management in children (51). Hypnosis has been proposed as a particularly appropriate intervention since children are generally more susceptible to hypnosis than adults (52). This increased susceptibility has been attributed to children's willingness to become absorbed in fantasy (53). The existing literature on hypnosis has been plagued by the lack of consensus over what procedures may be accurately described as 'hypnosis.' This lack of consistency is reflected in the variety of terms that have been used (e.g. hypnotherapy, guided imagery and imagery) to describe hypnotic techniques. For simplification, studies discussed in this section will be referred to as 'hypnosis' regardless of the terminology employed by the study authors, with the exception of interventions for recurrent pediatric headache (see below). It should be noted, however, that use of a single term to refer to these procedures does not necessarily indicate that the techniques employed across studies were comparable.

\section{Procedural Pain}

\section{Pediatric oncology}

To date, most pain-related hypnosis research in children has focused on the management of acute procedural pain, particularly in cancer patients. In their recent, comprehensive review, Wild and Espie (54) discuss nine studies conducted on the effects of hypnosis on painful medical procedures that pediatric oncology patients repeatedly undergo: lumbar puncture (LP) and bone marrow aspiration (BMA), procedures that have been rated by children as the two most painful and distressing procedures associated with cancer treatment (55). Following guidelines published by the Canadian Task Force on the Periodic Health Examination (56), Wild and Espie rated each study on a scale ranging from $1++$ (e.g. randomized controlled trials with very low risk of bias) to 4 (expert opinion). All of the studies except for three fell in the 2- category (i.e. case-control or cohort studies with a high risk of confounding or bias and a significant risk that the relationship is not causal). The three highest rated studies were ranked in the $2+$ category (i.e. well-conducted case-control or cohort studies with a low risk of confounding or bias and a moderate possibility that the relationship is causal).

Only these three studies (57-59) rated highest by Wild and Espie (54) included a control group. Notably, the findings 
across the three studies were not consistent. One study found no effects of hypnosis compared with cognitive-behavioral coping skills in alleviating pain and distress during BMA in thirty patients (age 5-15 years) (58), whereas another study found that hypnosis was significantly more effective than distraction in reducing distress, pain and anxiety during venipuncture, BMA and LP in highly hypnotizable children (age 3-8 years) (59). The third study found a small effect for hypnotic 'imaginative involvement', over distraction and standard medical care on procedural pain and distress during BMAs in younger (3-6 years) but not in older children (7-10 years) (57). Thus, Wild and Espie (54) assigned an overall grade of D to the existing body of evidence for use of hypnosis for procedural pain in pediatric oncology. This recommendation indicated that existing research has generated inconsistent results and was of generally poor methodological quality. The authors also highlighted several difficulties that made it impossible for them to compare results across studies or make firm decisions regarding the efficacy of the approaches used, including wide variation in: (i) ages of the children studied; (ii) outcome measures; and (iii) hypnotic techniques or procedures used.

In a study that was not included in the review by Wild and Espie (54), Liossi and colleagues (60) conducted a randomized controlled trial testing the efficacy of a manual-based clinical hypnosis intervention in alleviating pain in 80 pediatric cancer patients (6-16 years of age) undergoing regular LP. Patients received either one of two forms of hypnosis, attention control or standard medical care. The hypnosis groups (which were equally effective) reported less pain and anxiety and showed less behavioral distress than controls. However, improvements degraded when patients were switched to self-hypnosis. The authors concluded that hypnosis is effective in preparing pediatric oncology patients for LP, but the presence of the therapist may be critical. Methodologically, this study is superior to prior studies in that a treatment manual was used to guide the intervention and adherence checks for treatment fidelity were conducted by an independent observer. However, there was only one therapist in this study and it is unclear whether treatment effects would generalize to other therapists. When this study is taken into account, we deviate somewhat from Wild and Espie (54) in designating hypnosis as a possibly efficacious treatment for pediatric procedural pain in oncology patients.

\section{Non-cancer patients}

In two studies with samples comprised of mostly adult patients, hypnosis was shown to be superior to an attention control and no treatment in reducing pain during dressing change $(61,62)$. However, in the only published study by Foertsch and colleagues (63) that specifically examined the impact of hypnosis on pediatric burn victims, hypnosis was no better than attention control. In this study, 23 children (aged 3-12 years) were randomly assigned to receive 'familiar imagery' treatment (i.e. imagery related to familiar experiences) or social support control (i.e. casual chat) during three dressing changes. Behavioral ratings of distress were conducted by trained raters unaware of group assignment. Hypnosis did not result in decreased distress in the treated group relative to baseline, nor were there any differences between the treated and control groups. The authors maintain that several factors may be responsible for the null findings: (i) all the children studied were in-patients whereas out-patients are typically studied; (ii) the length of the burn dressing change procedure is highly variable compared with other medical procedures; and (iii) the age of the children studied, while representative of pediatric burn victims, was younger than the average of children studied for other medical procedures. Nevertheless, based on the above results, it is unclear whether hypnosis holds promise as an intervention for pain related to pediatric burn dressing procedures.

\section{Post-operative pain}

Hypnosis or guided imagery has been used in at least two published studies on post-operative pain in children $(64,65)$. In one study, 52 children (aged 7-19 years) were randomly assigned to a single session of hypnosis which included suggestions for favorable post-operative outcomes $\sim 1$ week prior to surgery or standard care (65). The hypnosis group evidenced significantly lower post-operative pain ratings and shorter length of hospital stays compared with controls, although the groups did not differ on anxiety or the amount of pain medication received. A recent, rigorously conducted study by Huth and colleagues (64) randomly assigned 73 children (aged 7-2 years) to: (i) a treatment condition involving the viewing of a videotape on the use of imagery and then listening to a 30 min audiotape of imagery $\sim 1$ week prior to surgery (T1); or (ii) an attention control group. The treatment group also listened to the audiotape 1-4 h after surgery (T2) and 22-27 h after discharge at home (T3). The control group received standard care, including an equal amount of pre-operative attention as the experimental group. The imagery group reported less pain and anxiety at T2 than controls, after trait anxiety and post-surgery analgesic intake were controlled for, although there were no group differences in pain or anxiety at $\mathrm{T} 3$.

This study by Huth et al. (64) was methodologically superior to prior research since it included standardized administration of the intervention via videotapes and audiotapes. The main limitation of this study was lack of a sham treatment for the control group. The authors maintained that neither use of a tape with white noise nor simply waiting for the 30 min hypnosis interval was feasible since children may have distracted themselves in some other way (e.g. watching TV). Nevertheless, the findings of this study support the designation of possibly efficacious for hypnosis for post-operative pediatric pain.

\section{Chronic Pain}

\section{Recurrent pediatric headache}

In their review of ESTs for recurrent pediatric headache, Holden and colleagues (66) included 11 studies testing the 
efficacy of relaxation/self-hypnosis/guided imagery (referred to below as 'relaxation') for recurrent pediatric headaches. Of the four studies which included an appropriate control group (67-70), two studies independently found that 'relaxation' was superior to placebo control $(68,70)$, whereas the other two studies did not find support for the superiority of 'relaxation' over placebo $(67,69)$. The review mentions three other studies that found that 'relaxation' was superior to self-monitoring $(71,72)$, including one multiple baseline study (73). Follow-up data from these studies generally indicated maintenance of treatment gains across various post-treatment intervals (66).

Holden et al. (66) also reviewed two randomized, double-blind, crossover studies that compared relaxation/self-hypnosis with medications for recurrent pediatric headache $(74,75)$. The first study (74) compared home-based 'relaxation' with prophylactic pharmacotherapy in 48 adolescents with tension headaches. The results indicated significant improvement following 'relaxation', with no further gains associated with additional pharmacotherapy, although the degree of clinical improvement was modest. The second study (75) compared propranolol, placebo and self-hypnosis in 28 pediatric migraine patients (aged 6-12 years). The results indicated that self-hypnosis significantly reduced the frequency but not the intensity of headaches compared with propranolol, although Holden et al. pointed out that the drug washout periods were relatively short, thereby limiting the utility of these results.

Holden and colleagues (66) thus concluded that there was sufficient evidence to classify relaxation/self-hypnosis/guided imagery as well established and efficacious for recurrent pediatric migraine and tension headaches. However, it is unclear whether their conclusions may be extrapolated to all forms of hypnosis, particularly as it appears that self-hypnosis/guided imagery was typically included as part of an overall 'relaxation training' package in many of the studies reviewed. Thus, it is not known whether self-hypnosis by itself is sufficient to promote therapeutic change. It also appears that self-hypnosis may be more effective in certain patients than in others. For example, Liossi et al. (60) found that treatment gains were degraded when pediatric oncology patients were switched from therapist-assisted hypnosis to self-hypnosis for LP. On the other hand, self-hypnosis appears to be equally effective as clinic-based approaches for pediatric recurrent headache (66). When considering the total body of evidence, it is also worth noting the study by Foertsch et al. (63) which found no beneficial effects for hypnosis in pediatric burn victims during dressing change, as well as the conclusions of Wild and Espie (54) who maintained that evidence for the efficacy of hypnosis for procedural pain in pediatric oncology was relatively poor and inconsistent. As mentioned above, one of the difficulties in evaluating this literature concerns the lack of consensus over what constitutes hypnosis. Thus, future studies should consider the use of treatment manuals, as was done by Liossi et al. (60), to help ensure the comparability of procedures across studies. As previously discussed, the APA Task Force criteria require the use of a treatment manual for an intervention to qualify as an EST.

\section{Massage}

\section{Chronic Pain}

Only one published study has specifically examined the impact of massage on chronic pain in children (76). In this study, 20 children with juvenile rheumatoid arthritis (JRA) aged 5-14 years received either a daily $15 \mathrm{~min}$ massage by their parents or a daily $15 \mathrm{~min}$ relaxation session with their parents. At the conclusion of the 30 day trial, the massage group experienced less pain according to both child and parent report compared with controls. A physician who was unaware of group assignment also rated the massage group as having less pain and less morning stiffness. Strengths of this study include use of a standardized massage procedure and assessments by an independent physician. On the other hand, several methodological problems limit the study's utility. In addition to the small sample size, it is unclear whether patients were randomly assigned to study groups. Whereas teaching parents to massage their children appears to be a feasible and cost-effective way to conduct the intervention, it is not known how well parents adhered to the massage protocol or how often they performed the massage. It is also questionable whether relaxation constituted an appropriate control group for the 'touch' part of the intervention, since there was no physical contact involved. Comparison with a more appropriate control such as sham massage (light touch) would permit investigation of specific effects pertaining to massage while controlling for non-specific effects due to physical contact. Measurement of relaxation effects would also help determine if use of relaxation provided a control for that aspect of massage therapy.

\section{Procedural Pain}

The only other controlled study of massage for children's pain examined distress during dressing changes in pediatric burn patients (77). Prior to dressing changes, 24 children (mean age $=2.5$ years) were randomly assigned to receive either massage therapy or attention control (i.e. casual chat with the therapist). Children in the massage group received a $15 \mathrm{~min}$ massage from a trained therapist conducted according to a standardized protocol, applied to areas of the body that were not burned. Independent raters unaware of group assignment rated children's distress before and during the procedure. Massage patients evidenced minimal distress behaviors (aside from an increase in torso movements), whereas the control group showed increased facial grimacing, crying, torso movement, leg movement and reaching out. These results support the beneficial effects of massage for procedural pain in pediatric burn patients, although comparison with an appropriate control group (i.e. that controlled for physical contact) and repeated assessments across multiple procedures would increase confidence in the findings.

In sum, massage therapy appears to be a promising intervention for pediatric pain symptoms, although larger scale, randomized controlled trials incorporating a sham massage condition are needed before massage may be considered an 
EST. Work by Field and colleagues (76,78-80) has demonstrated the feasibility of using a standardized massage protocol. Nevertheless, use of detailed treatment manuals as well as ratings of treatment adherence would substantially advance the quality of research in this area.

\section{Conclusions and Future Directions: Additional Work is Needed to Establish Efficacy of CAM Approaches for Pediatric Pain}

A summary of the evidence supporting the CAM modalities discussed above is displayed in Table 1. In general, most of the CAM interventions reviewed are included in the promising or possibly efficacious categories. The only intervention (self-hypnosis/relaxation/guided imagery for recurrent pediatric headaches) that may be considered efficacious is actually a combined package of interventions. Thus, the efficacy of self-hypnosis/guided imagery in isolation remains unclear. As is evident from the preceding discussion, the quality of the studies examining the efficacy of CAM interventions for pain in children varies widely. Only two of the interventions, BFB and hypnosis, have a relatively substantial body of work supporting their application, although these are limited to a

Table 1. Summary of empirical evidence for efficacy of CAM interventions for pediatric pain

\begin{tabular}{|c|c|c|}
\hline CAM modality & Condition & Designation \\
\hline Acupuncture & $\begin{array}{l}\text { Chronic pain (various) } \\
\text { Pediatric migraine }\end{array}$ & $\begin{array}{l}\text { Promising } \\
\text { Possibly efficacious }\end{array}$ \\
\hline $\begin{array}{l}\text { Biofeedback } \\
\text { Thermal } \\
\text { biofeedback } \\
\text { EMG-biofeedback }\end{array}$ & $\begin{array}{l}\text { Pediatric migraine } \\
\text { Tension headaches } \\
\text { Tension headaches }\end{array}$ & $\begin{array}{l}\text { Possibly efficacious }^{\mathrm{a}} \\
\text { Promising } \\
\text { Promising }^{\mathrm{a}}\end{array}$ \\
\hline $\begin{array}{l}\text { Creative arts } \\
\text { Music }\end{array}$ & Injection pain & Promising \\
\hline $\begin{array}{l}\text { Herbal therapy } \\
\text { Naturopathic } \\
\text { extract } \\
\text { Peppermint oil }\end{array}$ & $\begin{array}{l}\text { Ear pain }(\mathrm{AOM}) \\
\text { Irritable bowel syndrome }\end{array}$ & $\begin{array}{l}\text { Promising } \\
\text { Possibly efficacious }\end{array}$ \\
\hline \multicolumn{3}{|l|}{ Homeopathy } \\
\hline & Ear pain $(\mathrm{AOM})$ & Promising \\
\hline \multirow[t]{4}{*}{ Hypnosis } & $\begin{array}{l}\text { Procedural pain in pediatric } \\
\text { oncology }\end{array}$ & Possibly efficacious \\
\hline & $\begin{array}{l}\text { Procedural pain in pediatric } \\
\text { burn injuries }\end{array}$ & Unclear \\
\hline & Post-operative pain & Possibly efficacious \\
\hline & Recurrent pediatric headache & Efficacious $^{\mathrm{b}}$ \\
\hline \multirow[t]{2}{*}{ Massage therapy } & Juvenile rheumatoid arthritis & Promising \\
\hline & $\begin{array}{l}\text { Procedural pain in pediatric } \\
\text { burn injuries }\end{array}$ & Promising \\
\hline
\end{tabular}

$\mathrm{AOM}=$ acute otitis media; Designation = designation according to criteria for empirically supported therapies (ESTs) by the American Psychological Association (APA) Division 12 Task Force on Promotion and Dissemination of Psychological Procedures.

${ }^{\mathrm{a}}$ Recommendation of Hermann and Blanchard (22).

${ }^{b}$ Recommendation of Holden et al. (66) for the category of relaxation/selfhypnosis/guided imagery/autogenic training. relatively circumscribed set of pain problems. The remaining interventions reviewed have been the subject of very few controlled investigations. Even within those CAM modalities that have amassed a number of empirical reports examining treatment outcome, the quality of the methodology across studies varied a great deal. Thus, conclusions regarding designations according to APA Task Force criteria in this review may not be considered definitive. It should also be noted that some of the designations in Table 1 were derived from other reviews, and interpretation of the APA Task Force criteria may have varied slightly across reviewers.

Generally speaking, research on CAM interventions for pediatric pain would benefit from a more systematic approach to achieving EST status. As discussed by Wild and Espie (54), many studies have skipped several steps in the EST process by comparing an unproven intervention with an already established treatment (e.g. cognitive-behavioral approaches) without first showing that the intervention is superior to a no-treatment control or standard medical care. Thus, they recommend that the first step in securing EST status might involve large-scale, randomized studies, perhaps drawing from multiple settings in order to provide sufficient sample sizes, comparing the CAM intervention with a no-intervention or 'care as usual' condition (54). Only if the results of such studies show that the intervention yields benefits exceeding that of standard care or nointervention should further work be undertaken to compare the intervention with established therapies and/or an attention control/placebo condition. Another important consideration is isolating the therapeutic ingredient(s) that lead to change. Given that many CAM interventions have been studied within the context of an overall treatment package that includes many different treatment components, careful treatment dismantling studies testing the efficacy of each individual component should also be conducted if the overall package is shown to be efficacious.

Several additional recommendations may be incorporated into future work. First, for interventions that do not easily lend themselves to testing against a 'pill placebo' or equivalent placebo formulation or placebo procedure (e.g. acupuncture), standardization of the intervention procedures using a treatment manual or its equivalent is a requirement for EST designation. The majority of studies have not used a manual, making it difficult to compare results across studies and to replicate results by independent research groups. Use of a manual will also allow assessments of the degree of adherence to the treatment protocol on the part of therapists. The lack of standardization appears to be particularly problematic in the hypnosis literature, which has a relatively large body of studies dating back $>20$ years; this lack of standardization is reflected in the disparate terms used to describe hypnotic procedures (e.g. guided imagery and hypnotherapy). Secondly, increased attention should be paid to methodological confounds that may lead positive findings to be attributable to non-specific effects, rather than specific effects of the intervention. Among the most important considerations include: (i) random group assignment; (ii) ensuring that both researchers and patients are kept unaware of group assignment (i.e. the equivalent of the 
'double-blind' pharmacological trial); (iii) use of valid and reliable outcome measures; (iv) including sufficient sample sizes to achieve adequate statistical power to detect between-group differences; and (v) use of an appropriate control condition. For certain interventions such as music therapy, inclusion of a placebo group (e.g. other auditory stimuli) to control for nonspecific effects is particularly important. Finally, even though mechanisms of action in CAM interventions may not be well understood, systematic testing of existing mechanistic models should be pursued (54). Hermann and Blanchard (22) pointed out that if the beneficial effects of BFB on pain are found to be mediated by psychological (e.g. self-efficacy) rather than physiological (e.g. muscle tension) factors, this would increase the likelihood that BFB will be studied in relation to other pain problems (e.g. RAP) rather than headache alone.

In sum, there are few high-quality empirical investigations that permit definitive conclusions to be drawn regarding the efficacy of CAM interventions for pediatric pain. The studies to date do, however, provide many useful findings that may guide researchers in conducting more carefully controlled investigations. It should be noted that several CAM interventions for pediatric pain were not included in this review due to a lack of published, controlled studies. These interventions include movement therapies such as yoga, other interventions involving the creative arts such as art or dance therapy, as well as meditation, energy healing, aromatherapy, folk remedies and spiritual approaches. This list is not exhaustive. Notably, many of these CAM treatments have shown encouraging findings in case reports and uncontrolled studies. It is our hope that future work may be directed at the careful testing of these unproven but potentially therapeutic CAM modalities. The pursuit of safe, efficacious and cost-effective interventions for pain in children that may be used in place of, or in concert with, conventional medical approaches is a worthy goal that should be undertaken with the highest degree of scientific rigor.

\section{References}

1. Eisenberg DM, Kessler RC, Foster C, Norlock FE, Calkins DR, Delbanco TL. Unconventional medicine in the United States: prevalence, costs, and patterns of use. N Engl J Med 1993;328:246-52.

2. Davis MP, Darden PM. Use of complementary and alternative medicine by children in the United States. Arch Pediatr Adolesc Med 2003;157: 393-6.

3. Ottolini MC, Hamburger EK, Loprieato JO, Coleman RH, Sachs HC, Madden R et al. Complementary and alternative medicine use among children in the Washington, DC area. Ambul Pediatr 2001;1:122-5.

4. Simpson N, Pearce A, Finlay F, Lenton S. The use of complementary medicine in paediatric outpatient clinics. Ambul Child Health 1998;3: $351-6$.

5. Ernst E. Prevalence of complementary/alternative medicine for children: a systematic review. Eur J Pediatr 1999;158:7-11.

6. Grootenhuis MA, Last BF, de Graaf-Nijkerk JH, van der Wel M. Use of alternative treatment in pediatric oncology. Cancer Nurs 1998;21:282-8.

7. Stern RC, Canda ER, Doershuk CF. Use of nonmedical treatment by cystic fibrosis patients. J Adolesc Health 1992;13:612-5.

8. Astin JA. Why patients use alternative medicine: results of a national study. J Am Med Assoc 1998;279:1548-53.

9. Bausell RB, Lee W, Berman BM. Demographic and health-related correlates of visits to complementary and alternative medical providers. Med Care 2001;39:190-6.
10. Eisenberg DM, Davis RB, Ettner SL, Appel S, Wilkey S, Van Rompay M et al. Trends in alternative medicine use in the United States, 1990-1997. J Am Med Assoc 1998;280:1569-75.

11. Chambless DL, Hollon SD. Defining empirically supported therapies. J Consult Clin Psychol 1998;66:7-18.

12. Task Force on Promotion and Dissemination of Psychological Procedures. Training in and dissemination of empirically validated psychological treatments: report and recommendations. Clin Psychol 1995;48:3-23.

13. Melchart D, Linde K, Fischer P, White A, Allais G, Vickers A et al. Acupuncture for recurrent headaches: a systematic review of randomized controlled trials. Cephalalgia 1999;19:779-86; discussion 765.

14. Ernst E, White AR. Acupuncture for back pain: a meta-analysis of randomized controlled trials. Arch Intern Med 1998;158:2235-41.

15. Ernst E, White A. Life-threatening adverse reactions after acupuncture? A systematic review. Pain 1997;71:123-6.

16. MacPherson H. Fatal and adverse events from acupuncture: allegation, evidence, and the implications. J Altern Complement Med 1999;5:47-56.

17. Kemper KJ, Sarah R, Silver-Highfield E, Xiarhos E, Barnes L, Berde C. On pins and needles? Pediatric pain patients' experience with acupuncture. Pediatrics 2000;105:941-7.

18. Zeltzer LK, Tsao JCI, Stelling C, Powers M, Levy S, Waterhouse M. A phase I study on the feasibility of an acupuncture/hypnotherapy intervention for chronic pediatric pain. J Pain Symptom Manag 2002;24:437-46.

19. Pintov S, Lahat E, Alstein M, Vogel Z, Barg J. Acupuncture and the opioid system: implications in management of migraine. Pediatr Neurol 1997; 17:129-33.

20. Baldi E, Salmon S, Anselmi B, Spillantini MG, Cappelli G, Brocchi A et al. Intermittent hypoendorphinaemia in migraine attack. Cephalalgia 1982;2:77-81.

21. Leone M, Sacerdote P, D’Amico D, Panerai AE, Bussone G. Beta-endorphin concentrations in the peripheral blood mononuclear cells of migraine and tension-type headache patients. Cephalalgia 1992;12:154-7.

22. Hermann C, Blanchard EB. Biofeedback in the treatment of headache and other childhood pain. Appl Psychophysiol Biofeedback 2002;27:143-62.

23. Scharff L, Marcus DA, Masek BJ. A controlled study of minimal-contact thermal biofeedback treatment in children with migraine. J Pediatr Psychol 2002;27:109-19.

24. Hernandez-Latorre MA, Roig M. Natural history of migraine in childhood. Cephalalgia 2000;20:573-9.

25. Grazzi L, Leone M, Frediani F, Bussone G. A therapeutic alternative for tension headache in children: treatment and 1-year follow-up results. Biofeedback Self Regul 1990;15:1-6.

26. Kroner-Herwig B, Mohn U, Pothmann R. Comparison of biofeedback and relaxation in the treatment of pediatric headache and the influence of parent involvement on outcome. Appl Psychophysiol Biofeedback 1998; 23:143-57.

27. Kroner-Herwig B, Plump U, Pothmann R. Progressive muscle relaxation und EMB-Biofeedback in der therapie von chronischem kopfschmerz bein kindern. Der Schmerz 1992;6:121-7.

28. Bussone G, Grazzi L, D’Amico D, Leone M, Andrasik F. Biofeedbackassisted relaxation training for young adolescents with tension-type headache: a controlled study. Cephalalgia 1998;18:463-7.

29. Arndorfer RE, Allen KD. Extending the efficacy of a thermal biofeedback treatment package to the management of tension-type headaches in children. Headache 2001;41:183-92.

30. Lavigne JV, Ross CK, Berry SL, Hayford JR, Pachman LM. Evaluation of a psychological treatment package for treating pain in juvenile rheumatoid arthritis. Arthritis Care Res 1992;5:101-10.

31. Cozzi L, Tryon WW, Sedlacek K. The effectiveness of biofeedbackassisted relaxation in modifying sickle cell crises. Biofeedback Self Regul 1987;12:51-61.

32. Sundaram R. Art therapy with a hospitalized child. Am J Art Ther 1995; $34: 2-8$.

33. Barrera ME, Rykov MH, Doyle SL. The effects of interactive music therapy on hospitalized children with cancer: a pilot study. Psychooncology 2002;11:379-88.

34. Favara-Scacco C, Smirne G, Schiliro G, Di Cataldo A. Art therapy as support for children with leukemia during painful procedures. Med Pediatr Oncol 2001;36:474-80.

35. Arntz A, Dreessen L, Merckelbach H. Attention, not anxiety, influences pain. Behav Res Ther 1991;29:41-50.

36. Farthing GW, Venturino M, Brown SW. Suggestion and distraction in the control of pain: test of two hypotheses. J Abnorm Psychol 1984;93: 266-76. 
37. Fowler-Kerry S, Lander JR. Management of injection pain in children. Pain 1987;30:169-75.

38. Megel ME, Houser CW, Gleaves LS. Children's responses to immunizations: lullabies as a distraction. Issues Compr Pediatr Nurs 1999;21: 129-45.

39. Malone AB. The effects of live music on the distress of pediatric patients receiving intravenous starts, venipunctures, injections, and heel sticks. J Music Ther 1996;33:19-33.

40. Aitken JC, Wilson S, Coury D, Moursi AM. The effect of music distraction on pain, anxiety and behavior in pediatric dental patients. Pediatr Dent 2002;24:114-8.

41. Arts SE, Abu-Saad HH, Champion GD, Crawford MR, Fisher RJ, Juniper KH et al. Age-related response to lidocaine-prilocaine (EMLA) emulsion and effect of music distraction on the pain of intravenous cannulation. Pediatrics 1994;93:797-801.

42. Marchette L, Main R, Redick E, Bagg A, Leatherland J. Pain reduction interventions during neonatal circumcision. Nurs Res 1991;40:241-4.

43. Sarrell EM, Mandelberg A, Cohen HA. Efficacy of naturopathic extracts in the management of ear pain associated with acute otitis media. Arch Pediatr Adolesc Med 2001;155:796-9.

44. Sarrell EM, Cohen HA, Kahan E. Naturopathic treatment for ear pain in children. Pediatrics 2003;111:e574-9.

45. Rosenfeld RM. What to expect from medical treatment of otitis media. Pediatr Infect Dis J 1995;14:731-7; quiz 738 .

46. Lehnert T. Acute otitis media in children. Role of antibiotic therapy. Can Fam Physician 1993;39:2157-62.

47. Ghelardini C, Galeotti N, Salvatore G, Mazzanti G. Local anaesthetic activity of the essential oil of Lavandula angustifolia. Planta Med 1999; 65:700-3.

48. Kline RM, Kline JJ, Di Palma J, Barbero GJ. Enteric-coated, pH-dependent peppermint oil capsules for the treatment of irritable bowel syndrome in children. J Pediatr 2001;138:125-8.

49. Weydert JA, Ball TM, Davis MF. Systematic review of treatments for recurrent abdominal pain. Pediatrics 2003;111:e1-11.

50. Jacobs J, Springer DA, Crothers D. Homeopathic treatment of acute otitis media in children: a preliminary randomized placebo-controlled trial. Pediatr Infect Dis J 2001;20:177-83.

51. Olness K, Gardner G. Hypnosis and Hypnotherapy with Children. Philadelphia: Grune \& Stratton, 1988.

52. Morgan A, Hilgard E. Age differences in susceptibility to hypnosis. Int J Clin Exp Hypn 1973;21:78-85.

53. Gardner GG. Hypnosis with children. Int J Clin Exp Hypn 1974;22: 20-38.

54. Wild MR, Espie CA. The efficacy of hypnosis in the reduction of procedural pain and distress in pediatric oncology: a systematic review. J Dev Behav Pediatr 2004;25:207-13.

55. McGrath PJ, Hsu E, Cappelli M, Luke B. Pain from pediatric cancer: a survey of an outpatient oncology clinic. J Psychosocial Oncol 1990;8: 109-24.

56. Canadian Task Force on the Periodic Health Examination. The Periodic Health Examination, 1979.

57. Kuttner L, Bowman M, Teasdale M. Psychological treatment of distress, pain, and anxiety for young children with cancer. J Dev Behav Pediatr 1988;9:374-81.

58. Liossi C, Hatira P. Clinical hypnosis versus cognitive behavioral training for pain management with pediatric cancer patients undergoing bone marrow aspirations. Int J Clin Exp Hypn 1999;47:104-16.

59. Smith JT, Barabasz A, Barabasz M. Comparison of hypnosis and distraction in severely ill children undergoing painful medical procedures. J Counseling Psychol 1996;43:187-95.
60. Liossi C, Hatira P. Clinical hypnosis in the alleviation of procedurerelated pain in pediatric oncology patients. Int J Clin Exp Hypn 2003;51: 4-28.

61. Patterson DR, Everett JJ, Burns GL, Marvin JA. Hypnosis for the treatment of burn pain. J Consult Clin Psychol 1992;60:713-7.

62. Patterson DR, Questad KA, de Lateur BJ. Hypnotherapy as an adjunct to narcotic analgesia for the treatment of pain for burn debridement. Am J Clin Hypn 1989;31:156-63.

63. Foertsch CE, O'Hara MW, Stoddard FJ, Kealey GP. Treatment-resistant pain and distress during pediatric burn-dressing changes. J Burn Care Rehabil 1998;19:219-24.

64. Huth MM, Broome ME, Good M. Imagery reduces children's postoperative pain. Pain 2004;110:439-48.

65. Lambert SA. The effects of hypnosis/guided imagery on the postoperative course of children. J Dev Behav Pediatr 1996;17:307-10.

66. Holden EW, Deichmann MM, Levy JD. Empirically supported treatments in pediatric psychology: recurrent pediatric headache. J Pediatr Psychol 1999;24:91-109.

67. Emmen HH, Passchier J. Treatment of headache among children by progressive relaxation. Cephalalgia 1987;7 Suppl 6:387-9.

68. Larsson B, Melin L. Chronic headaches in adolescents: treatment in a school setting with relaxation training as compared with informationcontact and self-registration. Pain 1986;25:325-36.

69. McGrath PJ, Humphreys P, Goodman JT, Keene D, Firestone P, Jacob P et al. Relaxation prophylaxis for childhood migraine: a randomized placebo-controlled trial. Dev Med Child Neurol 1988;30:626-31.

70. McGrath PJ, Humphreys P, Keene D, Goodman JT, Lascelles MA, Cunningham SJ et al. The efficacy and efficiency of a self-administered treatment for adolescent migraine. Pain 1992;49:321-4.

71. Larsson B, Daleflod B, Hakansson L, Melin L. Therapist-assisted versus self-help relaxation treatment of chronic headaches in adolescents: a school-based intervention. J Child Psychol Psychiatry 1987;28:127-36.

72. Larsson B, Melin L, Lamminen M, Ullstedt F. A school-based treatment of chronic headaches in adolescents. J Pediatr Psychol 1987;12:553-66.

73. Engel JM. Relaxation training: a self-help approach for children with headaches. Am J Occup Ther 1992;46:591-6.

74. Larsson B, Melin L, Doberl A. Recurrent tension headache in adolescents treated with self-help relaxation training and a muscle relaxant drug. Headache 1990;30:665-71.

75. Olness K, MacDonald JT, Uden DL. Comparison of self-hypnosis and propranolol in the treatment of juvenile classic migraine. Pediatrics 1987; 79:593-7.

76. Field T, Hernandez-Reif M, Seligman S, Krasnegor J, Sunshine W, RivasChacon $\mathrm{R}$ et al. Juvenile rheumatoid arthritis: benefits from massage therapy. J Pediatr Psychol 1997;22:607-17.

77. Hernandez-Reif M, Field T, Largie S, Hart S, Redzepi M, Nierenberg B et al. Childrens' distress during burn treatment is reduced by massage therapy. J Burn Care Rehabil 2001;22:191-5; discussion 190 .

78. Field TM. Touch therapies. In: Hoffman RR, Sherrick MF, Warm JS, editors. Viewing Psychology As a Whole. Washington, DC: American Psychological Association, 1998, 603-24.

79. Field TM. Massage therapy effects. Am Psychol 1998;53:1270-81.

80. Field TM, Sunshine W, Hernandez-Reif $M$ et al. Chronic fatigue syndrome: massage therapy effects on depression and somatic symptoms in chronic fatigue syndrome. J Chronic Fatigue Syndr 1997;3:43-51.

Received on January 11, 2005; accepted on April 7, 2005 


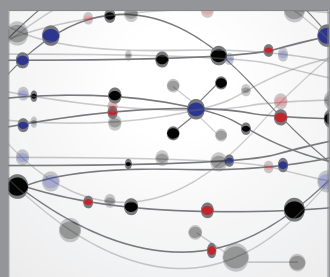

The Scientific World Journal
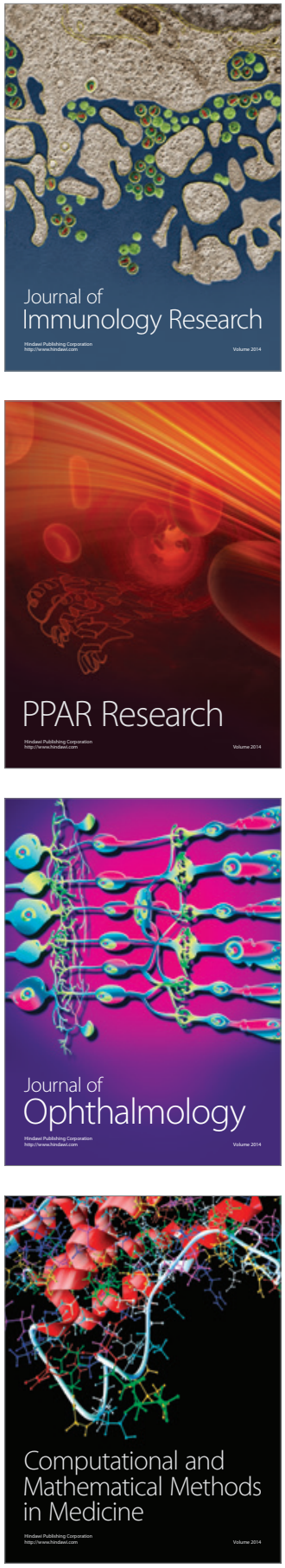

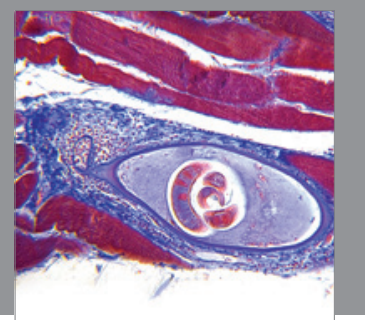

Gastroenterology

Research and Practice
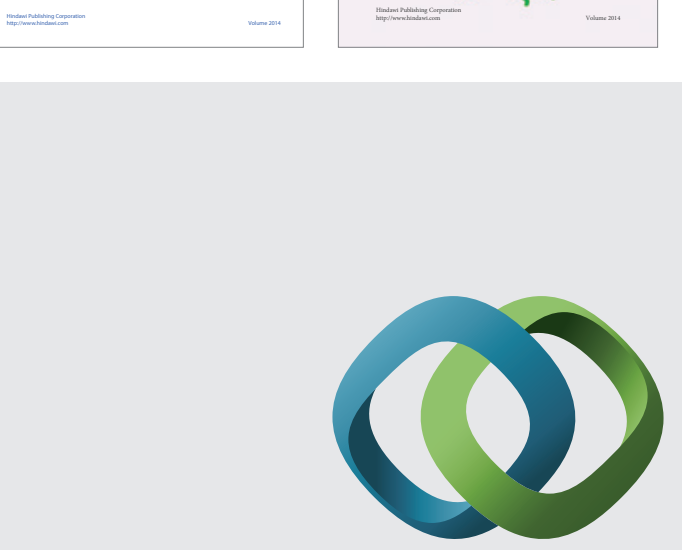

\section{Hindawi}

Submit your manuscripts at

http://www.hindawi.com
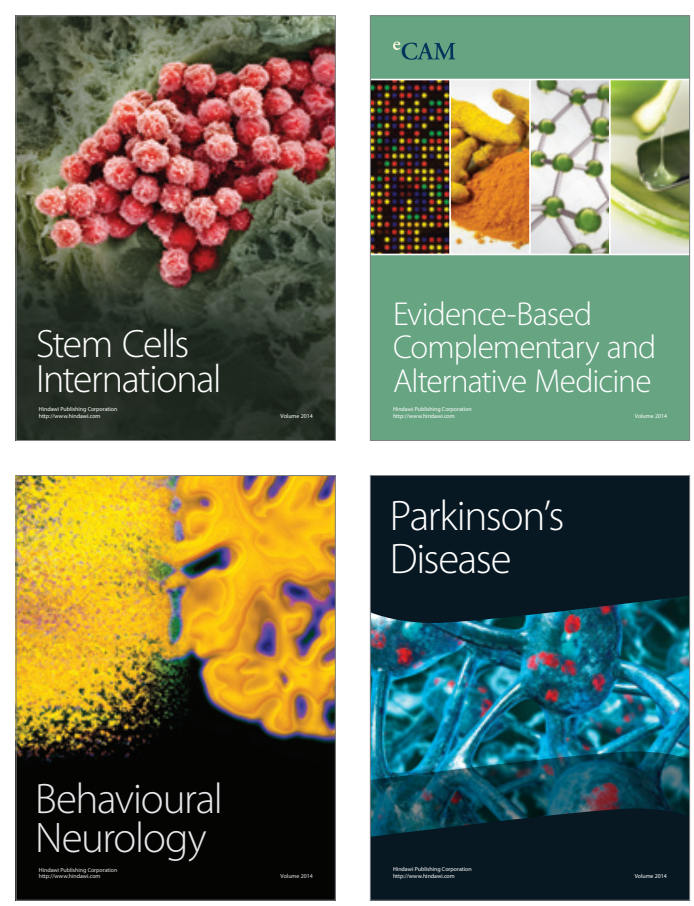

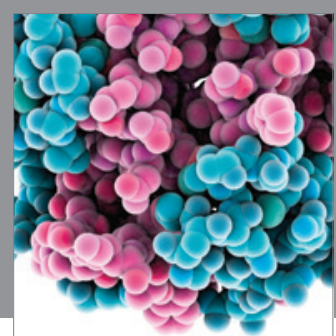

Journal of
Diabetes Research

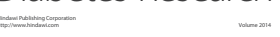

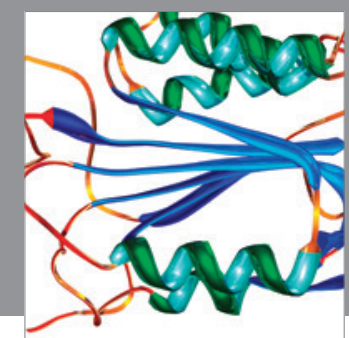

Disease Markers
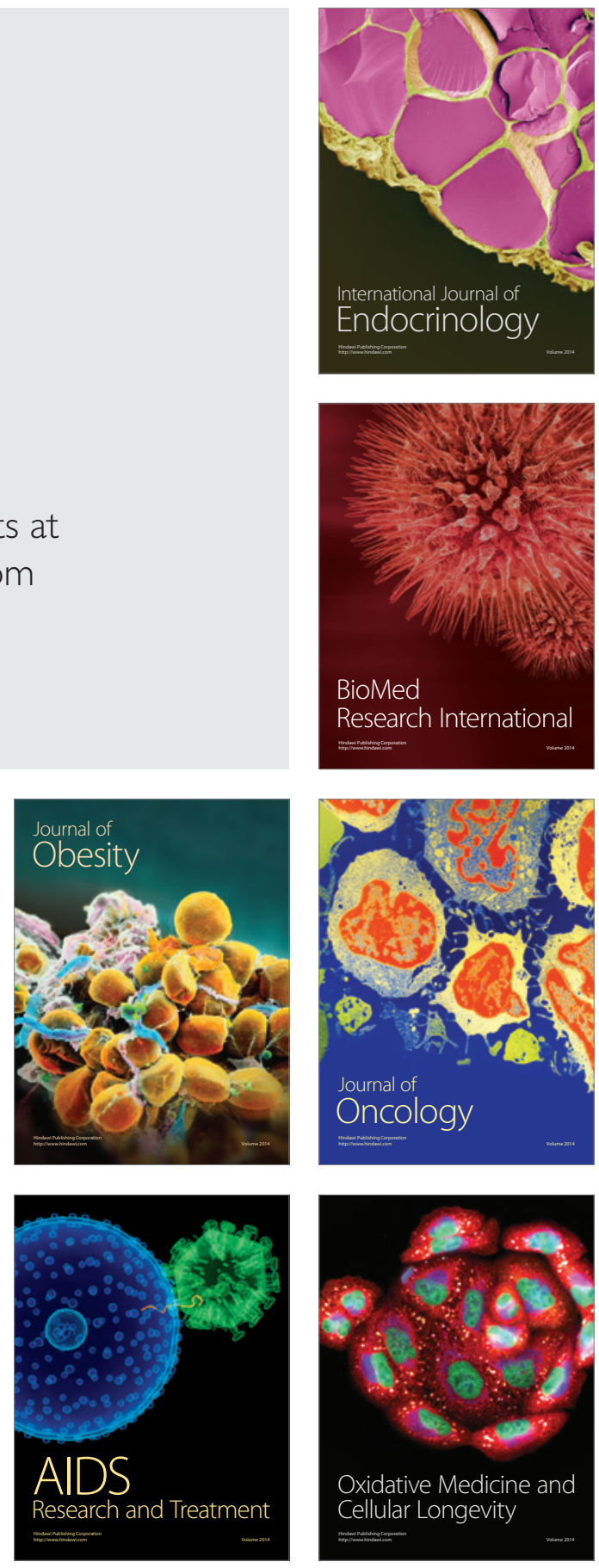